\title{
Prevalence of elevated mean arterial pressure and how fitness moderates its association with $\mathrm{BMl}$ in youth
}

\author{
Ayodele A Ogunleye ${ }^{1, *}$, Gavin R Sandercock ${ }^{1}$, Christine Voss ${ }^{1}$, Joey C Eisenmann ${ }^{2,3}$ \\ and Katharine Reed ${ }^{1}$ \\ ${ }^{1}$ Centre for Sport and Exercise Science, School of Biological Sciences, University of Essex, Wivenhoe Campus, \\ Colchester, Essex, CO4 3SQ, UK: ${ }^{2}$ Department of Pediatrics and Human Development, Michigan State University, \\ East Lansing, MI, USA: ${ }^{3}$ Department of Kinesiology, Michigan State University, East Lansing, MI, USA
}

Submitted 20 December 2011: Final revision received 9 July 2012: Accepted 24 August 2012: First published online 19 0ctober 2012

\begin{abstract}
Objective: Cardiorespiratory fitness is known to be cardioprotective and its association with the components of the metabolic syndrome in children is becoming clearer. The aim of the present study was to examine the extent to which cardiorespiratory fitness may offset the weight-related association with mean arterial pressure (MAP) in schoolchildren.

Design: Cross-sectional study.

Settings: Schoolchildren from the East of England, UK.

Subjects: A total of 5983 (48\% females) schoolchildren, 10 to 16 years of age, had height, weight and blood pressure measured by standard procedures and cardiorespiratory fitness assessed by the $20 \mathrm{~m}$ shuttle-run test. Participants were classified as fit or unfit using internationally accepted fitness cut-off points; and as normal weight, overweight or obese based on BMI, again using international cut-off points. Age-adjusted ANCOVA was used to determine the main effects and interaction of fitness and BMI on MAP Z-score. Logistic regression models were used to estimate odds ratios of elevated MAP.

Results: Prevalence of elevated MAP in schoolchildren was $14 \cdot 8 \%$ overall and $35.7 \%$ in those who were obese-unfit. Approximately $21 \%$ of participants were overweight and 5\% obese, while $23 \%$ were classified as unfit. MAP generally increased across BMI categories and was higher in the aerobically unfit participants. Obese-fit males had lower MAP compared with obese-unfit males $(P<0 \cdot 001)$; this trend was similar in females $(P=0 \cdot 05)$.

Conclusions: Increasing fitness level may have a positive impact on the weightrelated elevations of MAP seen in obese and overweight schoolchildren.
\end{abstract}

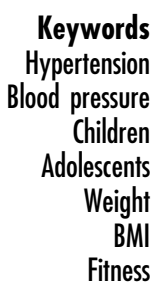

The associations between cardiorespiratory fitness, hypertension, all-cause mortality and chronic disease are well established in adults ${ }^{(1,2)}$. Research shows that cardiorespiratory fitness (fitness) attenuates the negative health consequences of high adiposity (fatness) in adults ${ }^{(3)}$ due to the so-called 'fat but fit' phenomenon ${ }^{(4,5)}$.

Reported secular declines in childhood fitness ${ }^{(6)}$ and increases in adiposity are both alarming and well documented in many developed countries including the $\mathrm{UK}^{(7)}$. As body weight is positively associated with blood pressure $(\mathrm{BP})^{(8)}$, it is unsurprising that a secular increase in children's BP has also been reported ${ }^{(9)}$. In schoolchildren, that cardiorespiratory fitness is cardioprotective may be well established $^{(10-12)}$, but details of how it interacts with body composition to mediate BP remain inconsistent.

Lee et $a l^{(5)}$ examined this 'fit but fat' hypothesis in 21925 males (aged 30-83 years). Mortality rates after 8 years of follow-up showed that those who were fit enjoyed protection from the potential deleterious health effects of being overweight. Fitness has also been shown to attenuate metabolic risk independent of abdominal $\operatorname{adiposity}^{(13)}$. Stevens et $a l^{(14)}$, on the other hand, found no significant interaction between fitness and fatness as predictors of all-cause or cardiovascular mortality. Their study did, however, confirm the efficacy of cardiorespiratory fitness in reducing mortality.

Cardiorespiratory fitness and adiposity are interdependent and controversy remains as to the relative independence of relationships between fitness and fatness in youth. One source of controversy may be the interchange between physical activity and cardiorespiratory fitness as effect modifiers; it is important to note that fitness and physical activity are distinct constructs. Further controversy likely stems from the use of different estimates and cut-off points for fatness (percentage body fat, BMI or waist circumference), the often arbitrary definitions of 
cardiorespiratory fitness ${ }^{(11,15)}$ and the study of samples of different age groups ${ }^{(16,17)}$. Arbitrary cut-off points such as median split of age-adjusted BMI, physical working capacity and values of standardized residuals have been used in previous studies ${ }^{(15,18)}$ rather than more preferable, criterion-referenced classifications.

We attempted to address these deficiencies by using accepted cut-off points for cardiorespiratory fitness and BMI. First, we defined BMI as normal weight, overweight or obese using internationally defined cut-off points based on predicted adult values of $<25,25-30$ or $>30 \mathrm{~kg} / \mathrm{m}^{2}$, respectively ${ }^{(19)}$. We then defined low fitness using a retro-extrapolated cut-off point which predicts an adult value below the 20th percentile and an increased risk of cardiomyopathy ${ }^{(20)}$. Finally, we assessed how fitness and BMI interact with one another and their subsequent associations with BP in youth. We hypothesized that high levels of fitness would attenuate the relationship between overweight/obesity and BP. Our secondary aim was to evaluate the prevalence of elevated mean arterial pressure (MAP) in English schoolchildren.

\section{Materials and methods}

\section{Study population}

A total of 5983 schoolchildren aged 10-16 years were recruited from a structured convenience sample of twenty-three state-run, comprehensive schools. All data were collected between 2007 and 2009. We sent letters to schools in the East of England region inviting them to participate and from responders we purposefully selected a sample designed to have characteristics similar to the East of England's population in terms of rural $(30 \%)$ or urban dwelling (70\%) and area-level deprivation. All participants were enrolled in the ongoing East of England Healthy Hearts Study. Pupils who normally attended physical education classes were included in the study. Using the exclusion criteria of the presence of known CVD or a lack of parental or pupil consent, we achieved a response rate of $>98 \%$ for the measurements made in the present study ( $2 \%$ of available pupils were withdrawn). The study was approved by the University of Essex Ethics Committee.

\footnotetext{
Antbropometry

We measured stature to the nearest $1 \mathrm{~mm}$ (Seca Leicester Height Measure; Seca GmbH \& Co. KG, Hamburg, Germany) and mass to the nearest $0 \cdot 1 \mathrm{~kg}$ (Seca 888 digital scale; Seca $\mathrm{GmbH} \& \mathrm{Co} . \mathrm{KG}$ ) with participants dressed in T-shirt and shorts, without shoes. BMI was calculated $\left(\mathrm{kg} / \mathrm{m}^{2}\right)$, converted to $Z$-scores based on UK reference data ${ }^{(19)}$ and categorized as normal weight, overweight or obese according to International Obesity Taskforce (IOTF) criteria ${ }^{(19)}$. Data required for BMI categorization were missing for 104 participants, reducing the sample size to $n 5879$.
}

\section{Cardiorespiratory fitness}

Cardiorespiratory fitness was assessed using the $20 \mathrm{~m}$ shuttle-run test (20mSRT) administered in the form of the FITNESSGRAM PACER, a modified version of the original protocol $^{(21)}$. Participants had previously taken part in the 20mSRT as part of their physical education. Participants were encouraged by both the instructions on the PACER $\mathrm{CD}$ and a researcher to 'run for as long as possible'. The test requires volunteers to run back and forth over a marked distance of $20 \mathrm{~m}$ in time with an audible signal. The test starts at an initial running speed of $8.0 \mathrm{~km} / \mathrm{h}$ and increases initially by $1 \mathrm{~km} / \mathrm{h}$ after the first minute and then by $0.5 \mathrm{~km} / \mathrm{h}$ each minute thereafter. Researchers acted as 'spotters' and recorded the final shuttle count at either the point of volitional exhaustion or when the participant failed to maintain the required running speed twice. Final shuttle count was converted first to final running speed and then into $Z$-scores based on global performance indices ${ }^{(22)}$. $\mathrm{VO}_{2 \max }(\mathrm{ml} / \mathrm{kg}$ per min) was predicted based on final running speed and age ${ }^{(23)}$. FITNESSGRAM PACER Healthy Fitness Zone cut-offs ${ }^{(20)}$ were used to categorize participants. If participants' total completed shuttle count was above their age- and sex-specific cut-off, they were classed as 'fit'; otherwise they were classified as 'unfit'.

\section{Blood pressure measurement}

All BP measurements were carried out after the participant completed a physical activity questionnaire, which typically took 10-12 min. BP was measured after a further 5 min of quiet, seated rest. Trained researchers fitted an appropriately sized inflatable cuff around the upper left arm of each participant. Participants were instructed to sit still in their chair with their left arm resting on a table at the same level as the heart. Two measures of systolic blood pressure (SBP) and diastolic blood pressure (DBP) were made using an automated sphygmomanometer (Omron MX3; Omron Healthcare Europe BV, Hoofddorp, The Netherlands). The lowest measures of SBP and DBP were recorded, since the first reading in a series of BP measurements is typically higher when oscillometric devices are employed ${ }^{(24,25)}$. All BP measurements were taken before the fitness test. Participants' MAP values (in $\mathrm{mmHg}$ ) were generated from SBP and DBP values using the following formula:

$$
\mathrm{MAP}=\frac{2}{3}(\mathrm{DBP})+\frac{1}{3}(\mathrm{SBP}) .
$$

Standardized scores were derived for MAP ( $Z$-score) using the $\mathrm{BP}$ reference charts for the $\mathrm{UK}^{(8)}$, which adjust for age, sex and skewness.

\section{Deprivation quintile}

We obtained an area-level measure of deprivation for each participant using home postcode. The English Index of Multiple Deprivation 2007 (IMD 2007) is measured based on the small-area geographical units known as lower super output areas, details of which have been 
described elsewhere ${ }^{(26)}$. Within the present data, IMD 2007 scores ranged from 1.96 to $62 \cdot 5$. Quintiles of deprivation were generated from the ranked IMD 2007 scores in which the first quintile (1) represented the least deprived and the last quintile (5) the most deprived.

\section{Data analysis}

Participants were grouped by fitness (either fit or unfit) ${ }^{(20)}$ and BMI category (normal weight, overweight or obese) ${ }^{(19)}$. ANCOVA, controlling for age, was used to determine the main effects and interaction of fitness and BMI category on MAP Z-score. Post hoc analyses were performed using the Bonferroni multiple comparison test. Data were analysed separately for males and females, due to the differences between sexes in BMI, fitness and MAP. Since sex may also be an important determinant of health and illness, analysing data by sex is encouraged ${ }^{(27)}$.

Logistic regression analysis was conducted to calculate odds ratios of elevated MAP from categorical fitness and fatness variables. MAP centiles were generated from ageand sex-specific $Z$-scores and participants were classified as having high or low MAP using the 91st centile cut-off as recommended $^{(8)}$. Predictors in the regression analysis were weight status (BMI category) and fitness (fit, unfit). In a second model the interaction between BMI category and fitness was also examined. All statistical analyses were performed using the statistical software package PASW Statistics 18.

\section{Results}

Participants' SBP, DBP and MAP according to age, gender, BMI category and fitness are shown in Table 1 . The overall prevalence of elevated MAP was $15 \%$, but reached $36 \%$ in obese schoolchildren who were also unfit. There was a significant difference in MAP between sexes $(P<0 \cdot 001)$. MAP increased according to age and was higher in the overweight and obese BMI categories as well as in unfit participants $(P<0 \cdot 001)$. There were no significant between-sex or between-age differences after converting MAP to $Z$-scores $(P>0 \cdot 05)$.

Overall, $21 \%$ of the sample was overweight and $5 \%$ obese. In total, $23 \%$ of the sample was classified as unfit; this was less common in normal weight (16\%) than in either overweight (35\%) or obese (63\%) participants. The combined influence of fitness and BMI category on MAP is shown separately for males and females in Fig. 1. The MAP $Z$-score of fit-obese schoolchildren was significantly lower than that of unfit-obese ones $(P<0 \cdot 001)$. The difference in MAP $Z$-score between fit-overweight and unfitoverweight schoolchildren was not significant $(P>0 \cdot 05)$. In fit participants, $\mathrm{VO}_{2 \max }$ was $48 \cdot 2(\mathrm{sD} 6 \cdot 1) \mathrm{ml} / \mathrm{kg}$ per min (males) and $43 \cdot 8(\mathrm{sD} 4 \cdot 7) \mathrm{ml} / \mathrm{kg}$ per $\min$ (females), compared with $47.9(\mathrm{sD} 5 \cdot 3) \mathrm{ml} / \mathrm{kg}$ per min (males) and $39 \cdot 9$ (SD $2 \cdot 6$ ) $\mathrm{ml} / \mathrm{kg}$ per min (females) in unfit participants.
In males, there were main effects for BMI $(F=36 \cdot 64$, $P<0 \cdot 001$; MAP $Z$-score mean and SD: 0.33 (SD 0.85) in normal weight ( $n$ 2268); 0.61 (SD 0.83) in overweight ( $n$ 649); 0.87 (SD 0.87) in obese $(n$ 172)) and cardiorespiratory fitness $(F=12 \cdot 31, P<0 \cdot 001$; MAP $Z$-score mean and SD: 0.53 (SD 0.87) in unfit ( $n$ 831); 0.39 (sD 0.86) in fit ( $n$ 2283); Table 2). In females, there were main effects for BMI $(F=28 \cdot 83, P<0 \cdot 001$; MAP $Z$-score mean and SD: $0 \cdot 36$ (SD 0.82) in normal weight ( $n$ 2059); 0.64 (SD 0.80) in overweight ( $n 590) ; 0 \cdot 75$ (SD 0.79) in obese $(n 141))$, but not cardiorespiratory fitness $(F=0.96, P=0.33$; MAP $Z$-score mean and SD: $0 \cdot 51$ (SD 0.82) in unfit ( $n$ 535); $0 \cdot 43$ (SD 0.83) in fit $(n$ 2334)). There was a significant interaction between BMI and cardiorespiratory fitness in males $(F=7.54$, $P<0 \cdot 01)$, but not in females $(F=2 \cdot 39, P=0 \cdot 09)$. Overall, obese-fit males $(P<0 \cdot 001)$ and females $(P=0 \cdot 05)$ had a lower mean MAP $Z$-score than their obese-unfit counterparts (Table 2).

Unadjusted and adjusted odds ratios and 95\% confidence intervals of the binary logistic regression predicting elevated MAP ( $>91$ st centile) are presented in Table 3. When BMI categories (categorized as normal weight, overweight or obese by IOTF cut-off points) and fitness (dichotomized by FITNESSGRAM cut-off points) were entered into the model, BMI was a significant predictor of elevated MAP; but no other variables added significantly to the prediction of MAP. Obese schoolchildren were over three times more likely to have a high MAP than normal-weight schoolchildren (adjusted $\mathrm{OR}=3 \cdot 91,95 \%$ CI $2 \cdot 91,5 \cdot 27$ ). There was no statistically significant difference in the likelihood of elevated MAP between the two fitness categories after adjusting for BMI only, BMI and sex, or BMI, sex, age and deprivation. How the likelihood of elevated MAP differed between BMI categories is shown in Table 3.

The results of the combined prediction of BMI category and fitness on elevated MAP are shown in Table 4. Fitness significantly attenuated the elevation of MAP associated with BMI categories. In Table 4, there was a difference in risk of elevated MAP within fitness and fatness categories: (i) obese-unfit, overweight-unfit and normal weight-unfit; (ii) obese-unfit, overweight-unfit and normal weight-fit; and almost in (iii) obese-unfit, overweight-unfit, obese-fit, overweight-fit, normal weight-unfit and normal weight-fit.

There was a clear trend towards elevated MAP in obesefit participants compared with those who were normal weight but unfit (OR $=1 \cdot 54,95 \%$ CI $0 \cdot 97,2 \cdot 45)$. Table 4 shows that obese-fit participants were more likely to have elevated MAP than those who were normal weight-fit $(\mathrm{OR}=1 \cdot 75,95 \% \mathrm{CI} 1 \cdot 10,2 \cdot 79)$. These odds were not as pronounced as the increased likelihood of elevated MAP found in the obese-unfit group compared with the normal weight-fit group (OR $=3 \cdot 98,95 \%$ CI 2·92, 5·41). The likelihood of elevated MAP in normal weight-unfit participants was not significantly different from that in those who were normal weight-fit $(\mathrm{OR}=0 \cdot 87,95 \%$ CI $0 \cdot 67,1 \cdot 13)$. 
Table 1 Baseline characteristics of the study participants in relation to their mean arterial pressure: schoolchildren ( $n$ 5983) aged 10-16 years, East of England, 2007-2009

\begin{tabular}{|c|c|c|c|c|c|c|c|c|c|c|c|c|c|}
\hline & \multicolumn{2}{|c|}{ Frequency } & \multicolumn{2}{|c|}{$\mathrm{SBP}(\mathrm{mmHg})$} & \multicolumn{2}{|c|}{$\mathrm{DBP}(\mathrm{mmHg})$} & \multicolumn{2}{|c|}{ MAP (mmHg) } & \multicolumn{2}{|c|}{ MAP Z-score } & \multirow[b]{2}{*}{$P$ (difference in MAP Z-scores) } & \multicolumn{2}{|c|}{ Elevated MAP prevalence } \\
\hline & $n$ & $\%$ & Mean & SD & Mean & SD & Mean & SD & Mean & SD & & $\%$ & $95 \% \mathrm{Cl}$ (Fisher's exact) \\
\hline \multicolumn{14}{|l|}{ Sex } \\
\hline Female & 2869 & $48 \cdot 0$ & $114 \cdot 96$ & $11 \cdot 2$ & 68.04 & $8 \cdot 9$ & $83 \cdot 68$ & $8 \cdot 2$ & 0.43 & 0.9 & $0 \cdot 31$ & $14 \cdot 5$ & $13 \cdot 2,16 \cdot 8$ \\
\hline Male & 3114 & $52 \cdot 0$ & $116 \cdot 26$ & $12 \cdot 1$ & $66 \cdot 01$ & $9 \cdot 0$ & $82 \cdot 76$ & $8 \cdot 3$ & 0.45 & 0.8 & & $15 \cdot 2$ & $14 \cdot 0,16 \cdot 5$ \\
\hline \multicolumn{14}{|l|}{ BMI (IOTF)t, } \\
\hline Obese & 313 & $5 \cdot 3$ & $119 \cdot 32$ & $11 \cdot 9$ & $70 \cdot 45$ & $9 \cdot 0$ & $86 \cdot 72$ & $8 \cdot 1$ & $0 \cdot 82$ & 0.8 & & $29 \cdot 7$ & $24 \cdot 7,35 \cdot 1$ \\
\hline Overweight & 1239 & $21 \cdot 1$ & $117 \cdot 45$ & 11.9 & 68.57 & 8.5 & $84 \cdot 86$ & $7 \cdot 9$ & 0.62 & $0 \cdot 8$ & 0.001 & $20 \cdot 0$ & $17 \cdot 8,22 \cdot 4$ \\
\hline Normal weight & 4327 & $73 \cdot 6$ & $114 \cdot 77$ & 11.5 & $66 \cdot 21$ & $9 \cdot 0$ & $82 \cdot 40$ & $8 \cdot 2$ & 0.35 & 0.8 & & $12 \cdot 0$ & $11 \cdot 0,13 \cdot 0$ \\
\hline \multicolumn{14}{|l|}{ Fitness } \\
\hline Fit & 4617 & $77 \cdot 2$ & $115 \cdot 57$ & $11 \cdot 6$ & $66 \cdot 63$ & $8 \cdot 9$ & $82 \cdot 94$ & $8 \cdot 2$ & 0.41 & $0 \cdot 8$ & $<0.001$ & $13 \cdot 9$ & $12 \cdot 9,14 \cdot 9$ \\
\hline Unfit & 1366 & $22 \cdot 8$ & $115 \cdot 88$ & $12 \cdot 0$ & $68 \cdot 19$ & $9 \cdot 0$ & 84.09 & $8 \cdot 2$ & 0.52 & 0.9 & & $18 \cdot 2$ & $16 \cdot 1,20 \cdot 3$ \\
\hline \multicolumn{14}{|l|}{ Age (years) $\dagger$} \\
\hline 10 & 270 & $4 \cdot 5$ & 111.09 & $11 \cdot 0$ & $66 \cdot 44$ & $10 \cdot 1$ & $81 \cdot 33$ & $9 \cdot 0$ & $0 \cdot 42$ & $1 \cdot 0$ & & $16 \cdot 7$ & $12 \cdot 4,21 \cdot 7$ \\
\hline 11 & 1097 & $18 \cdot 3$ & $112 \cdot 81$ & $10 \cdot 8$ & 66.09 & $9 \cdot 0$ & $81 \cdot 66$ & $8 \cdot 0$ & 0.38 & 0.9 & & $12 \cdot 3$ & $10 \cdot 4,14 \cdot 4$ \\
\hline 12 & 1749 & $29 \cdot 2$ & 114.05 & $11 \cdot 7$ & $66 \cdot 12$ & $9 \cdot 2$ & $82 \cdot 10$ & 8.5 & 0.37 & 0.9 & & $13 \cdot 3$ & $11 \cdot 8,15 \cdot 0$ \\
\hline 13 & 1337 & $22 \cdot 3$ & $116 \cdot 82$ & $11 \cdot 5$ & $67 \cdot 79$ & $8 \cdot 6$ & $84 \cdot 13$ & $7 \cdot 8$ & 0.51 & 0.8 & $<0.001$ & $16 \cdot 7$ & $14 \cdot 7,18 \cdot 8$ \\
\hline 14 & 980 & $16 \cdot 4$ & $117 \cdot 96$ & $11 \cdot 6$ & 67.98 & $8 \cdot 8$ & $84 \cdot 64$ & $8 \cdot 1$ & 0.48 & 0.8 & & $16 \cdot 7$ & $14 \cdot 5,19 \cdot 2$ \\
\hline 15 & 520 & $8 \cdot 7$ & $121 \cdot 38$ & $10 \cdot 9$ & $68 \cdot 16$ & $8 \cdot 3$ & $85 \cdot 90$ & $7 \cdot 5$ & 0.52 & 0.8 & & $16 \cdot 0$ & $12 \cdot 9,19 \cdot 4$ \\
\hline 16 & 30 & 0.5 & $124 \cdot 90$ & $11 \cdot 2$ & $66 \cdot 13$ & $9 \cdot 7$ & $85 \cdot 72$ & $8 \cdot 4$ & $0 \cdot 38$ & $0 \cdot 8$ & & $16 \cdot 7$ & $5 \cdot 6,34 \cdot 7$ \\
\hline Obese-unfit & 196 & $3 \cdot 3$ & $120 \cdot 28$ & $11 \cdot 5$ & $72 \cdot 12$ & $8 \cdot 6$ & $88 \cdot 18$ & $7 \cdot 6$ & 0.97 & 0.8 & & $35 \cdot 7$ & $29 \cdot 0,42 \cdot 9$ \\
\hline Overweight-unfit & 432 & $7 \cdot 4$ & $117 \cdot 32$ & $11 \cdot 8$ & $68 \cdot 79$ & $8 \cdot 1$ & $84 \cdot 97$ & $7 \cdot 4$ & 0.63 & 0.8 & & $20 \cdot 6$ & $16 \cdot 9,24 \cdot 7$ \\
\hline Normal weight-unfit & 702 & $11 \cdot 9$ & 113.55 & $11 \cdot 8$ & $66 \cdot 46$ & $9 \cdot 2$ & $82 \cdot 16$ & $8 \cdot 3$ & 0.31 & 0.8 & & $10 \cdot 8$ & $8 \cdot 6,13 \cdot 4$ \\
\hline Obese-fit & 117 & $2 \cdot 0$ & $117 \cdot 70$ & $12 \cdot 5$ & $67 \cdot 58$ & $9 \cdot 0$ & $84 \cdot 29$ & $8 \cdot 3$ & 0.56 & $0 \cdot 8$ & $<0.001$ & $19 \cdot 7$ & $12 \cdot 9,28 \cdot 0$ \\
\hline Overweight-fit & 805 & $13 \cdot 7$ & $117 \cdot 49$ & $11 \cdot 9$ & $68 \cdot 44$ & $8 \cdot 7$ & $84 \cdot 79$ & $8 \cdot 2$ & 0.62 & 0.8 & & $19 \cdot 6$ & $16 \cdot 9,22 \cdot 5$ \\
\hline Normal weight-fit & 3627 & $61 \cdot 7$ & $115 \cdot 01$ & $11 \cdot 4$ & $66 \cdot 17$ & $8 \cdot 9$ & $82 \cdot 45$ & $8 \cdot 2$ & 0.36 & 0.8 & & $12 \cdot 3$ & $11 \cdot 2,13 \cdot 4$ \\
\hline Total & 5983 & $100 \cdot 0$ & $115 \cdot 64$ & $11 \cdot 7$ & $66 \cdot 98$ & $9 \cdot 0$ & $83 \cdot 20$ & $8 \cdot 3$ & 0.44 & $0 \cdot 8$ & & $14 \cdot 8$ & $14 \cdot 0,15 \cdot 8$ \\
\hline
\end{tabular}

SBP, systolic blood pressure; DBP, diastolic blood pressure; MAP, mean arterial pressure; IOTF, International Obesity Taskforce.

$t P$ values of those variables with more than two groups (BMI and age) were derived from main effects, using ANOVA.

fBMI category missing for 104 participants $(1 \cdot 7 \%)$. 

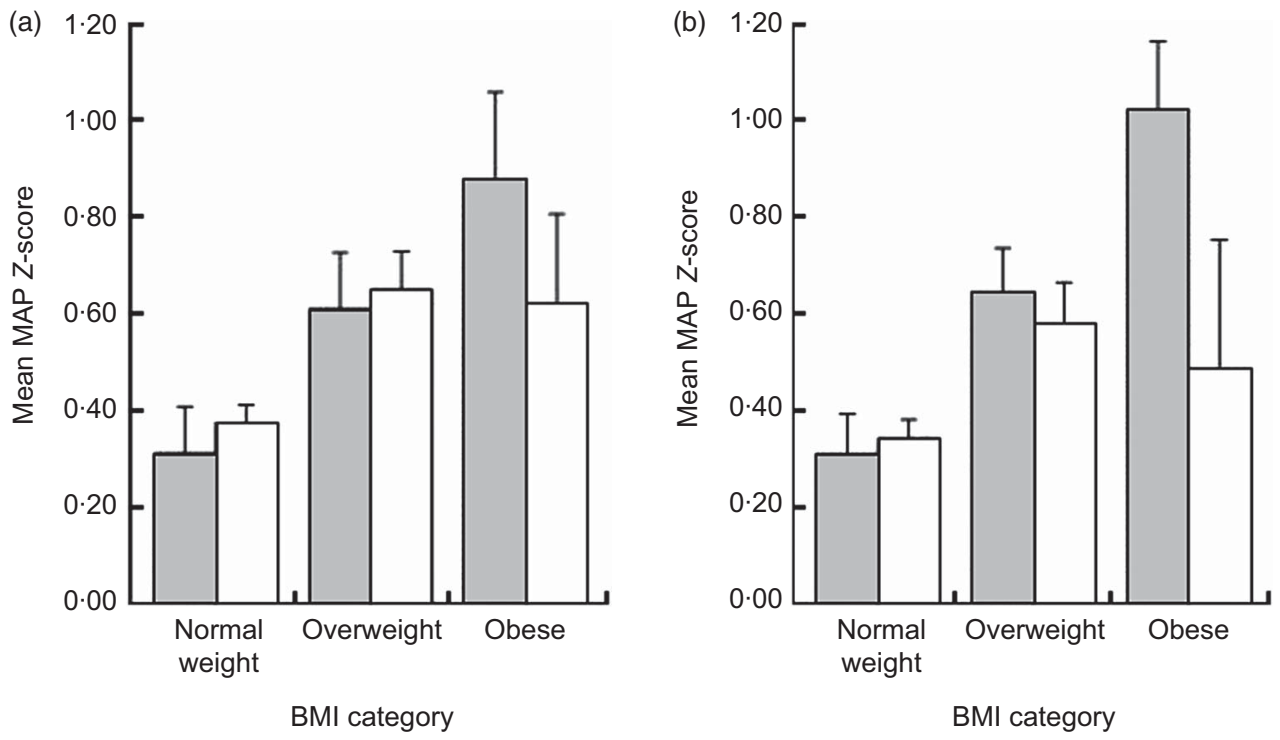

Fig. 1 Variation in mean arterial pressure (MAP) according to BMI category (normal weight, overweight and obese, using International Obesity Taskforce cut-offs) and fitness status ( $\square$, unfit; $\square$, fit) among schoolchildren ( $n$ 5983) aged $10-16$ years, East of England, 2007-2009: (a) females; (b) males. Values are means with $95 \%$ confidence intervals represented by vertical bars

Table 2 Bonferroni tests for multiple comparisons of mean differences and $95 \%$ confidence intervals of mean arterial pressure Z-scores among schoolchildren ( $n$ 5983) aged 10-16 years, East of England, 2007-2009

\begin{tabular}{|c|c|c|c|c|}
\hline \multirow[b]{3}{*}{ Multiple comparison } & \multicolumn{4}{|c|}{ MAP Z-score } \\
\hline & \multicolumn{2}{|c|}{ Females } & \multicolumn{2}{|c|}{ Males } \\
\hline & Mean difference & $95 \% \mathrm{Cl}$ & Mean difference & $95 \% \mathrm{Cl}$ \\
\hline \multicolumn{5}{|l|}{ BMI (IOTF)† } \\
\hline Normal weight $v$. overweight & $-0 \cdot 27^{\star \star}$ & $-0.36,-0.18$ & $-0 \cdot 28^{\star \star}$ & $-0.37,-0.19$ \\
\hline Normal weight $v$. obese & $-0 \cdot 39^{\star \star}$ & $-0.56,-0.22$ & $-0.54^{\star \star}$ & $-0.70,-0.38$ \\
\hline Overweight $v$. obese & $-0 \cdot 12^{N S}$ & $-0.30,0.07$ & $-0 \cdot 26^{\star *}$ & $-0.44,-0.09$ \\
\hline \multicolumn{5}{|l|}{ Fitnessł } \\
\hline Unfit $v$. fit & $0.08^{\mathrm{NS}}$ & $0.00,0 \cdot 16$ & $0 \cdot 15^{\star *}$ & $0.08,0.22$ \\
\hline \multicolumn{5}{|l|}{ Fitness in obese group } \\
\hline Unfit $v$. fit & $0.26^{\mathrm{NS}}$ & $0.00,0.52$ & $0.54^{\star *}$ & $0.27,0.82$ \\
\hline
\end{tabular}

MAP, mean arterial pressure; IOTF, International Obesity Taskforce.

${ }^{\star \star} P<0.01$.

tBMI category missing for 104 participants $(1 \cdot 7 \%)$.

‡Independent samples $t$ test was used for this analysis (Bonferroni test is appropriate only with $>3$ fitness groups).

\section{Discussion}

BMI and cardiorespiratory fitness are both associated with BP in youth, but assessing the independence of this relationship has been hampered by methodological differences between studies. We hypothesized that cardiorespiratory fitness would attenuate the adverse association that overweight and obesity have with BP in youth when both independent variables were classified using evidencebased cut-off points related to adult health outcomes. The results indicated that adequate levels of cardiorespiratory fitness may favourably modify the weight-related elevations in MAP observed in obese/overweight schoolchildren. Fitness did not however appear to have a significant impact on BP in normal-weight schoolchildren. There was a dose-response association between higher BMI and elevated MAP. Both overweight and obese schoolchildren who were fit had a reduced risk of elevated MAP than those of similar weight who were unfit.

MAP is derived from the combination of standard measures of SBP and DBP and is an important predictor of stroke ${ }^{(28,29)}$. MAP allows description of BP as a single measurement and is a robust tool suited to non-laboratory assessment protocols. We found similar results to those reported for MAP, when elevated ( $>91$ st centile) SBP and DBP were used as the outcome variable.

The prevalence of elevated MAP found in the present study is similar to that of others ${ }^{(30)}$. There are a number of previous studies which have tested the 'fit but fat' hypothesis ${ }^{(11,12,16,17)}$, although the present study is the first one in English schoolchildren. To our knowledge, the present study is the first with a sufficient sample size 
Table 3 Binary logistic regression output showing characteristics predicting elevated mean arterial pressure (>91st centile) among schoolchildren ( $n$ 5983) aged 10-16 years, East of England, 2007-2009

\begin{tabular}{|c|c|c|c|c|c|c|c|c|c|c|c|c|}
\hline \multirow[b]{2}{*}{ Predictor } & \multicolumn{4}{|c|}{ Combined } & \multicolumn{4}{|c|}{ Males } & \multicolumn{4}{|c|}{ Females } \\
\hline & Unadjusted OR & $95 \% \mathrm{Cl}$ & Adjusted ORt & $95 \% \mathrm{Cl}$ & Unadjusted OR & $95 \% \mathrm{Cl}$ & Adjusted OR & $95 \% \mathrm{Cl}$ & Unadjusted OR & $95 \% \mathrm{Cl}$ & Adjusted OR & $95 \% \mathrm{Cl}$ \\
\hline \multicolumn{13}{|l|}{ BMI (IOTF)‡ } \\
\hline Obese & $3 \cdot 10 \S$ & $2 \cdot 39,4 \cdot 02$ & $3 \cdot 91 \S$ & $2 \cdot 91,5 \cdot 27$ & $3.52 \S$ & $2 \cdot 51,4.95$ & $4 \cdot 30 \S$ & $2 \cdot 89,6 \cdot 38$ & $2 \cdot 57 \S$ & $1 \cdot 72,3 \cdot 86$ & $3 \cdot 30 \S$ & $2 \cdot 09,5 \cdot 22$ \\
\hline Overweight & $1 \cdot 84 \S$ & $1 \cdot 55,2 \cdot 17$ & $1.97 \S$ & $1 \cdot 65,2 \cdot 36$ & $1.63 \S$ & $1 \cdot 29,2 \cdot 05$ & $1 \cdot 77 \S$ & $1 \cdot 38,2 \cdot 28$ & $2 \cdot 07 \S$ & $1 \cdot 63,2 \cdot 64$ & $2 \cdot 19 \S$ & $1 \cdot 69,2 \cdot 84$ \\
\hline Normal weight & 1.00 & Ref. & 1.00 & Ref. & $1 \cdot 00$ & Ref. & $1.00^{\circ}$ & Ref. & 1.00 & Ref. & 1.00 & Ref. \\
\hline \multicolumn{13}{|l|}{ Fitness } \\
\hline Unfit & $1 \cdot 38 \S$ & $1 \cdot 17,1 \cdot 62$ & 1.02 & $0 \cdot 84,1 \cdot 23$ & $1 \cdot 42 \S$ & $1 \cdot 15,1 \cdot 76$ & 1.02 & $0 \cdot 80,1 \cdot 30$ & $1 \cdot 29$ & $1 \cdot 00,1 \cdot 66$ & 1.03 & $0 \cdot 76,1 \cdot 39$ \\
\hline Fit & 1.00 & Ref. & 1.00 & Ref. & $1 \cdot 00$ & Ref. & $1 \cdot 00$ & Ref. & $1 \cdot 00$ & Ref. & $1 \cdot 00$ & Ref. \\
\hline \multicolumn{13}{|l|}{ Sex } \\
\hline Female & 0.94 & $0.82,1.09$ & 0.94 & $0.81,1 \cdot 10$ & - & - & - & - & - & - & - & - \\
\hline Male & $1 \cdot 00$ & Ref. & $1 \cdot 00$ & Ref. & - & - & - & - & - & - & - & - \\
\hline \multicolumn{13}{|l|}{ Age (years) } \\
\hline 10 & 1.00 & $0 \cdot 36,2 \cdot 75$ & 0.82 & $0 \cdot 30,2 \cdot 28$ & 0.87 & $0.23,3.27$ & $0 \cdot 72$ & $0 \cdot 19,2 \cdot 72$ & $1 \cdot 09$ & $0 \cdot 22,5 \cdot 37$ & 0.85 & $0 \cdot 17,4 \cdot 24$ \\
\hline 11 & $0 \cdot 70$ & $0 \cdot 26,1 \cdot 86$ & 0.63 & $0.23,1.68$ & 0.67 & $0 \cdot 19,2 \cdot 37$ & 0.62 & $0 \cdot 17,2 \cdot 20$ & $0 \cdot 70$ & $0 \cdot 15,3 \cdot 32$ & 0.58 & $0 \cdot 12,2 \cdot 75$ \\
\hline 12 & 0.79 & $0.29,2.03$ & $0 \cdot 71$ & $0 \cdot 27,1 \cdot 88$ & 0.90 & $0 \cdot 26,3 \cdot 15$ & 0.89 & $0.25,3 \cdot 13$ & 0.62 & $0 \cdot 13,2 \cdot 92$ & 0.50 & $0 \cdot 10,2 \cdot 35$ \\
\hline 13 & 1.00 & $0 \cdot 38,2 \cdot 64$ & 1.05 & $0 \cdot 39,2 \cdot 79$ & $1 \cdot 13$ & $0.32,3.94$ & $1 \cdot 18$ & $0 \cdot 34,4 \cdot 14$ & 0.85 & $0.18,3.99$ & $0 \cdot 82$ & $0 \cdot 17,3 \cdot 88$ \\
\hline 14 & 1.00 & $0.37,2 \cdot 66$ & 1.06 & $0.40,2 \cdot 82$ & 1.04 & $0.30,3.63$ & $1 \cdot 12$ & $0.32,3.94$ & 0.94 & $0.20,4.44$ & 0.90 & $0 \cdot 19,4 \cdot 27$ \\
\hline 15 & 0.95 & $0.35,2.55$ & 0.83 & $0 \cdot 31,2 \cdot 24$ & $1 \cdot 18$ & $0 \cdot 33,4 \cdot 19$ & $1 \cdot 02$ & $0.28,3.66$ & 0.66 & $0 \cdot 14,3 \cdot 22$ & 0.53 & $0 \cdot 11,2 \cdot 62$ \\
\hline 16 & 1.00 & Ref. & $1 \cdot 00$ & Ref. & 1.00 & Ref. & 1.00 & Ref. & $1 \cdot 00$ & Ref. & $1 \cdot 00$ & Ref. \\
\hline \multicolumn{13}{|l|}{ Deprivation (quintile) } \\
\hline 1 (least deprived) & 0.90 & $0 \cdot 71,1 \cdot 13$ & 0.94 & $0 \cdot 75,1 \cdot 19$ & 0.82 & $0 \cdot 60,1 \cdot 12$ & 0.86 & $0 \cdot 62,1 \cdot 19$ & 1.02 & $0 \cdot 72,1 \cdot 44$ & $1 \cdot 11$ & $0.77,1.58$ \\
\hline 2 & $0 \cdot 81$ & $0.64,1.02$ & 0.83 & $0.66,1.06$ & 0.84 & $0.61,1 \cdot 15$ & 0.84 & $0 \cdot 61,1 \cdot 16$ & 0.85 & $0.59,1.20$ & 0.85 & $0.59,1 \cdot 24$ \\
\hline 3 & 0.85 & $0.68,1.08$ & 0.84 & $0.66,1.07$ & $0.68 \S$ & $0.49,0.94$ & $0.65 \S$ & $0.46,0.91$ & $1 \cdot 16$ & $0.83,1.62$ & $1 \cdot 17$ & $0.82,1.66$ \\
\hline 4 & 0.96 & $0 \cdot 76,1 \cdot 21$ & 0.95 & $0.75,1 \cdot 20$ & 0.98 & $0.72,1.32$ & 0.93 & $0.68,1.27$ & 1.08 & $0.77,1.51$ & 1.05 & $0.74,1.50$ \\
\hline 5 (most deprived) & 1.00 & Ref. & 1.00 & Ref. & 1.00 & Ref. & 1.00 & Ref. & 1.00 & Ref. & 1.00 & Ref. \\
\hline
\end{tabular}

IOTF, International Obesity Task Force; Ref., referent category.

tAdjusted model contains BMI, fitness, sex and age.

łBMl category missing for 104 partips, sex 
Table 4 Odds ratios and $95 \%$ confidence intervals of elevated mean arterial pressure ( $>91$ st centile) determined by the combined influence of BMI and cardiorespiratory fitness among schoolchildren ( $n 5983)$ aged 10-16 years, East of England, 2007-2009

\begin{tabular}{|c|c|c|c|c|c|c|}
\hline \multirow[b]{2}{*}{ Interaction term } & \multicolumn{2}{|c|}{ Combined } & \multicolumn{2}{|c|}{ Males } & \multicolumn{2}{|c|}{ Females } \\
\hline & OR & $95 \% \mathrm{Cl}$ & OR & $95 \% \mathrm{Cl}$ & OR & $95 \% \mathrm{Cl}$ \\
\hline \multicolumn{7}{|l|}{ BMI (IOTF) $+\times$ fitness } \\
\hline Obese $\times$ unfit & $3 \cdot 98 \ddagger$ & $2 \cdot 92,5 \cdot 41$ & $4 \cdot 28 \ddagger$ & $2 \cdot 90,6 \cdot 32$ & $3 \cdot 39 \neq$ & $2 \cdot 03,5 \cdot 67$ \\
\hline Overweight $\times$ unfit & $1 \cdot 86 \ddagger$ & $1 \cdot 44,2 \cdot 39$ & $1 \cdot 70 \ddagger$ & $1 \cdot 22,2 \cdot 37$ & $2 \cdot 08 \ddagger$ & $1 \cdot 40,3 \cdot 10$ \\
\hline Normal weight $\times$ unfit & $0 \cdot 87$ & $0 \cdot 67,1 \cdot 13$ & 0.93 & $0.67,1 \cdot 28$ & $0 \cdot 75$ & $0.49,1 \cdot 16$ \\
\hline Obese $\times$ fit & $1 \cdot 75 \ddagger$ & $1 \cdot 10,2 \cdot 79$ & 1.93 & $0.97,3.82$ & $1 \cdot 64$ & $0 \cdot 87,3 \cdot 12$ \\
\hline Overweight $\times$ fit & $1 \cdot 75 \ddagger$ & $1 \cdot 43,2 \cdot 14$ & $1 \cdot 54 \ddagger$ & $1 \cdot 15,2 \cdot 06$ & $1 \cdot 97 \ddagger$ & $1 \cdot 50,2 \cdot 60$ \\
\hline Normal weight $\times$ fit & $1 \cdot 00$ & Ref. & 1.00 & Ref. & $1 \cdot 00$ & Ref. \\
\hline
\end{tabular}

IOTF, International Obesity Task Force; Ref., referent category. tBMl category missing for 104 participants $(1 \cdot 7 \%)$.

$\ddagger$ Significantly different from the referent category.

to utilize the IOTF classifications. Participants in the European Youth Heart Study (EYHS) differed in fitness and fatness according to country of residence (Denmark, Portugal, Estonia and Norway) ${ }^{(12)}$; such was the influence of country on CVD risk factors that, when added as a dummy variable to prediction models for $\mathrm{BP}$, country had a greater influence than fitness. The participants in the current study had a higher prevalence of overweight, obesity and elevated BP than EYHS participants, due probably to our high inclusion rate and because English children tend to be fatter ${ }^{(31)}$.

\section{Fitness as an effect modifier}

In males, BMI was significantly associated with MAP and there was a difference in mean MAP $Z$-score between normal-weight and obese boys. Fitness had no influence on BP in normal-weight or overweight boys, but those who were obese-fit had significantly lower MAP than those who were obese-unfit. A similar trend was evident in females; obese-unfit girls had higher MAP than obesefit girls. When obese-fit and obese-unfit schoolchildren were compared with those who were normal weight-fit, there was a gradient in the prevalence of elevated MAP, with those who were obese-unfit having the highest prevalence of elevated MAP. The association between fatness and MAP was greatly modified by improving the specification of our regression model (including fitness and BMI interactions terms). In univariate regression analysis, fitness was a predictor of elevated MAP; however, fitness did not predict elevated MAP in a BMIadjusted model.

Previous studies have found differences in CVD risk factors in the most 'extreme' groups, i.e. high fat/low fit $v$. low fat/high fit, and vice versa ${ }^{(18,32)}$. Differences within 'fatness' categories like those shown here, are less common. It may be the case that we have found such differences in the present study due to separating participants into three clinically relevant 'fatness' categories (normal weight, overweight and obese), while other studies have used only two fatness categories (high fat/low fat) based on arbitrary cutoff points like median split ${ }^{(15,18,32)}$. The present data suggest a positive influence of fitness on MAP both in overweight and obese schoolchildren, which is more pronounced in the more markedly 'fat' (i.e. obese) when categorized using meaningful cut-off points. Our more rigorous methodology and use of agreed cut-off points have produced relatively novel findings with few comparable results from previous studies. For example, Eisenmann et al. ${ }^{(11)}$ found differences in MAP according to fitness/fatness category in children classified according to fatness (percentage body fat using recognized cut-off points ${ }^{(33)}$ ) and fitness (estimated oxygen consumption). Due to relatively small sample size they ${ }^{(11)}$ adjusted the fitness categories to ensure adequate participant numbers in each of the four fitness/fatness groups. Males with low fitness had significantly higher BP in those with high body fat compared with those with low body fat. Consistent with the present findings, high fitness was cardioprotective in girls with high body fat.

In the Quebec Family Study ${ }^{(18)}, 761$ children were classified into four BMI and fitness groups using median split. The authors then compared several CVD risk factors between groups. In agreement with our findings, unfit males and females had higher MAP than their fit counterparts within BMI categories.

The Aerobics Centre Longitudinal Study ${ }^{(32)}$ examined factors associated with the metabolic syndrome according to BMI category and fitness in 8-18-year-olds ( $n$ 484). In common with the present study, there were no differences in risk factors within the low BMI group according to fitness level but fitness did attenuate metabolic risk among overweight children and adolescents. Shaibi et $a l .{ }^{(34)}$ found univariate relationships between fitness and the metabolic syndrome in overweight youths, but these were non-significant after adjusting for fat mass. Like others ${ }^{(10)}$, they concluded that the association between fitness and metabolic health was a function of body composition. Overall, it appears that fatness plays a greater role than fitness in determining MAP and other metabolic risk factors. There is, however, a growing body of evidence that fitness can significantly attenuate the elevations in MAP observed in fatter children. This may be more apparent when obese children are studied as a 
distinct sub-sample as opposed to when a population is arbitrarily divided according to BMI.

The mechanisms linking BP with cardiorespiratory fitness in children are not fully understood. The benefits that cardiorespiratory fitness has on the vasculature are believed to be mediated by endothelial progenitor cells, which support vascular repair ${ }^{(35,36)}$. A healthy blood vessel requires an intact endothelium and a degree of elasticity. High fitness is associated with lower arterial stiffness and greater arterial compliance in children ${ }^{(37)}$ and may decrease total resistance ${ }^{(38)}$. There is also evidence, however, that vascular changes in response to physical training may be transitory ${ }^{(39)}$.

Given that the impact of interventions aimed at increasing physical activity on body composition has been negligible ${ }^{(40)}$, it is important to note that obese schoolchildren may achieve significant health benefits by improving their fitness, regardless of their BMI.

A limitation of the current study is that we were unable to adjust for biological maturity of the participants as no measure was available. Likewise we could not adjust for other confounding variables like diet, family history of hypertension and smoking status, all of which may be associated with MAP in youth.

Our measures of oscillometric BP were made in the 'field' and are comparable with office assessments made by physicians. They should not be regarded as comparable with clinical measures capable of diagnosing paediatric hypertension. They remain useful for inter-individual comparisons within the population but comparisons with values from other studies using different techniques and devices should be made with caution.

The cross-sectional nature of the study design limits our ability to draw causal inferences and prospective studies are needed to solve the issue of temporality. Despite the large sample size, the use of IOTF categories and the relatively low prevalence of elevated MAP in certain subgroups may limit the robustness of some of the regression analyses.

\section{Conclusions}

The present study is the first to analyse the combined influence of fitness and fatness on BP in English schoolchildren. Due to the large cohort of schoolchildren, we were able to use internationally recognized cut-off points for fitness and BMI in order to create meaningful and clinically relevant categories, which are a major improvement over previous studies. Although the results do not entirely show that high fitness eliminates the presence of elevated BP associated with high BMI, they do show that fitness weakens the direct association between BMI and elevated BP. It appears, however, that this attenuation may be limited to schoolchildren who are obese. Hypertension is one of the most prevalent co-morbidities associated with obesity in childhood ${ }^{(41)}$. Schoolchildren who are unfit and overweight or obese are at the highest risk of elevated BP. Given the stronger independent associations of simple objective measurements - cardiorespiratory fitness via field testing and BMI - with MAP, more emphasis should be placed on children's cardiorespiratory fitness and not only on BMI and weight reduction. The results suggest that obese individuals' MAP may be significantly improved by increasing fitness. Longitudinal studies are warranted to address the issue of temporality among these variables.

\section{Acknowledgements}

Sources of funding: The study was funded by the University of Essex through the Research Promotion Fund. Conflicts of interest: The authors declare no conflict of interest. Authors' contributions: A.A.O., G.R.S., C.V. and K.R. conceptualized the study and were involved in data collection in the schools. A.A.O. performed the regression analysis, and wrote parts of the Introduction and Results sections of the manuscript. K.R. performed the ANCOVA, wrote parts of the Introduction and Materials and Methods sections of the manuscript, and reviewed the manuscript. C.V. created the tables and figure. G.R.S. wrote the Discussion section of the manuscript. J.C.E. wrote part of the Introduction section of the manuscript, edited the Introduction, and proofread and reviewed the manuscript. Acknowledgements: The authors thank all persons who contributed to the development of this project, those who were involved in the data collection process in all schools, and all of the children and the Physical Education department staff of the schools for their participation.

\section{References}

1. Blair SN, Kohl HW 3rd, Barlow CE et al. (1991) Physical fitness and all-cause mortality in hypertensive men. Ann Med 23, 307-312.

2. Katzmarzyk PT, Church TS \& Blair SN (2004) Cardiorespiratory fitness attenuates the effects of the metabolic syndrome on all-cause and cardiovascular disease mortality in men. Arch Intern Med 164, 1092-1097.

3. Boule NG, Bouchard C \& Tremblay A (2005) Physical fitness and the metabolic syndrome in adults from the Quebec Family Study. Can J Appl Physiol 30, 140-156.

4. Duncan GE (2010) The 'fit but fat' concept revisited: population-based estimates using NHANES. Int J Behav Nutr Phys Act 7, 47.

5. Lee CD, Blair SN \& Jackson AS (1999) Cardiorespiratory fitness, body composition, and all-cause and cardiovascular disease mortality in men. Am J Clin Nutr 69, 373-380.

6. Tomkinson G \& Olds T (2007) Secular changes in pediatric aerobic fitness test performance: the global picture. Med Sports Sci 50, 46.

7. Ebbeling CB, Pawlak DB \& Ludwig DS (2002) Childhood obesity: public-health crisis, common sense cure. Lancet 360, 473-482.

8. Jackson L, Thalange N \& Cole T (2007) Blood pressure centiles for Great Britain. Arch Dis Child 92, 298-303. 
9. Din-Dzietham R, Liu Y, Bielo MV et al. (2007) High blood pressure trends in children and adolescents in national surveys, 1963 to 2002. Circulation 116, 1488-1496.

10. Eisenmann JC, Wickel EE, Welk GJ et al. (2005) Relationship between adolescent fitness and fatness and cardiovascular disease risk factors in adulthood: the Aerobics Center Longitudinal Study (ACLS). Am Heart J 149, 46-53.

11. Eisenmann JC, Welk GJ, Ihmels M et al. (2007) Fatness, fitness, and cardiovascular disease risk factors in children and adolescents. Med Sci Sports Exerc 39, 1251-1256.

12. Klasson-Heggebo L, Andersen LB, Wennlof AH et al. (2006) Graded associations between cardiorespiratory fitness, fatness, and blood pressure in children and adolescents. Br J Sports Med 40, 25-29.

13. Lee S, Kuk JL, Katzmarzyk PT et al. (2005) Cardiorespiratory fitness attenuates metabolic risk independent of abdominal subcutaneous and visceral fat in men. Diabetes Care 28, 895-901.

14. Stevens J, Cai J, Evenson KR et al. (2002) Fitness and fatness as predictors of mortality from all causes and from cardiovascular disease in men and women in the lipid research clinics study. Am J Epidemiol 156, 832-841.

15. Eisenmann JC, Welk GJ, Wickel EE et al. (2007) Combined influence of cardiorespiratory fitness and body mass index on cardiovascular disease risk factors among 8-18 year old youth: The Aerobics Center Longitudinal Study. Int J Pediatr Obes 2, 66-72.

16. Ruiz JR, Ortega FB, Loit HM et al. (2007) Body fat is associated with blood pressure in school-aged girls with low cardiorespiratory fitness: the European Youth Heart Study. J Hypertens 25, 2027-2034.

17. Nielsen GA \& Andersen LB (2003) The association between high blood pressure, physical fitness, and body mass index in adolescents. Prev Med 36, 229-234.

18. Eisenmann JC, Katzmarzyk PT, Perusse L et al. (2005) Aerobic fitness, body mass index, and CVD risk factors among adolescents: the Quebec family study. Int $J$ Obes (Lond) 29, 1077-1083.

19. Cole TJ, Bellizzi MC, Flegal KM et al. (2000) Establishing a standard definition for child overweight and obesity worldwide: international survey. BMJ 320, 1240-1243.

20. Leger LA \& Lambert J (1982) A maximal multistage 20-m shuttle run test to predict VO2 max. Eur J Appl Physiol Occup Physiol 49, 1-12.

21. Olds T, Tomkinson G, Leger L et al. (2006) Worldwide variation in the performance of children and adolescents: an analysis of 109 studies of the 20-m shuttle run test in 37 countries. J Sports Sci 24, 1025-1038.

22. Leger LA, Mercier D, Gadoury C et al. (1988) The multistage 20 metre shuttle run test for aerobic fitness. J Sports Sci $\mathbf{6}$, 93-101.

23. Meredith MD \& Welk GJ (2007) Interpreting FITNESSGRAM results. In FITNESSGRAM/ACTIVITYGRAM Administration Manual, 4th ed., pp. 59-68 [M Meredith and GJ Welk, editors]. Champaign, IL: Human Kinetics.
24. Gillman MW \& Cook NR (1995) Blood pressure measurement in childhood epidemiological studies. Circulation 92 , 1049-1057.

25. Park MK \& Menard SM (1989) Normative oscillometric blood pressure values in the first 5 years in an office setting. Am J Dis Child 143, 860-864.

26. Ogunleye AA, Voss C \& Sandercock GR (2012) Prevalence of high screen time in English youth: association with deprivation and physical activity. J Public Health (Oxf) 34, 46-53.

27. Anonymous (2011) Taking sex into account in medicine (Editorial). Lancet 378, 1826.

28. Zheng L, Sun Z, Li J et al. (2008) Pulse pressure and mean arterial pressure in relation to ischemic stroke among patients with uncontrolled hypertension in rural areas of China. Stroke 39, 1932-1937.

29. Verdecchia P, Schillaci G, Reboldi G et al. (2001) Different prognostic impact of 24-hour mean blood pressure and pulse pressure on stroke and coronary artery disease in essential hypertension. Circulation 103, 2579-2584.

30. McNiece KL, Poffenbarger TS, Turner JL et al. (2007) Prevalence of hypertension and pre-hypertension among adolescents. J Pediatr 150, 640-644, 644 e1.

31. International Obesity Taskforce (2012) World map of obesity. http://www.iaso.org/resources/world-map-obesity/?map = children (accessed September 2012).

32. Eisenmann JC (2007) Aerobic fitness, fatness and the metabolic syndrome in children and adolescents. Acta Paediatr 96, 1723-1729.

33. Eisenmann JC, Heelan KA \& Welk GJ (2004) Assessing body composition among 3- to 8-year-old children: anthropometry, BIA, and DXA. Obes Res 12, 1633-1640.

34. Shaibi GQ, Cruz ML, Ball GD et al. (2005) Cardiovascular fitness and the metabolic syndrome in overweight latino youths. Med Sci Sports Exerc 37, 922-928.

35. Steiner S, Niessner A, Ziegler S et al. (2005) Endurance training increases the number of endothelial progenitor cells in patients with cardiovascular risk and coronary artery disease. Atherosclerosis 181, 305-310.

36. Seals DR, Desouza CA, Donato AJ et al. (2008) Habitual exercise and arterial aging. J Appl Physiol 105, 1323-1332.

37. Reed KE, Warburton DE, Lewanczuk RZ et al. (2005) Arterial compliance in young children: the role of aerobic fitness. Eur J Cardiovasc Prev Rehabil 12, 492-497.

38. Pescatello LS, Franklin BA, Fagard R et al. (2004) American College of Sports Medicine position stand. Exercise and hypertension. Med Sci Sports Exerc 36, 533-553.

39. Watts K, Beye P, Siafarikas A et al. (2004) Effects of exercise training on vascular function in obese children. J Pediatr 144, 620-625.

40. Wareham NJ, van Sluijs EM \& Ekelund U (2005) Physical activity and obesity prevention: a review of the current evidence. Proc Nutr Soc 64, 229-247.

41. Sorof J \& Daniels S (2002) Obesity hypertension in children: a problem of epidemic proportions. Hypertension 40, 441-447. 